\title{
Introduction to volume 2, third issue
}

\section{Dear Colleagues,}

The current (7th) issue of JIG continues the tradition of publishing the papers presented at the Colorectal Cancer Symposium held at the Sacramento VAMC in March of 2012. March is National Colon Cancer Awareness Month in the United States. This one day symposium focused on methods to improve outcome of screening colonoscopy and training of endoscopists with a special emphasis on water-aided methods for colonoscopy. The symposium featured oral presentations, posters and live demonstration of water exchange colonoscopy. The topics published in the current issue include rapid communications on benchmarking and quality - screening colonoscopy, the water exchange method and difficult colonoscopy, the water exchange method for colonoscopy - effect of coaching; original studies on indigocarmine added to the water exchange method enhances adenoma detection - a RCT, a new method for screening and surveillance colonoscopy: combined water-exchange and cap-assisted colonoscopy, water exchange method for colonoscopy: learning curve of an experienced colonoscopist in a U.S. community practice setting, and the interim report of a randomized cross-over study comparing clinical performance of novice trainee endoscopists using conventional air insufflation versus warm water infusion colonoscopy. Critical commentaries of the above rapid communications and original studies are also provided by knowledgeable experts in the current issue. These include chromocolonoscopy for colorectal cancer screening: dive into the big blue, best of both the worlds - combining water-exchange and cap assisted colonoscopy, can old dogs learn new tricks, and re-learning colonoscopy: just a matter of time. An editorial that focuses on the issue: is colonoscopy best learned underwater, is also featured. An original study entitled magnetic endoscope Imaging (ScopeGuide) elucidates the mechanism of action of the painalleviating impact of water exchange colonoscopy - attenuation of loop formation, completes the discussion. 